\title{
A Novel Signature Derived from Metabolism-Related Genes GPT and SMS to Predict Prognosis of Laryngeal Squamous Cell Carcinoma
}

\section{Yujie Shen}

Fudan University Eye Ear Nose and Throat Hospital

\section{Qiang Huang}

Fudan University Eye Ear Nose and Throat Hospital

\section{Yifan Zhang}

Fudan University Eye Ear Nose and Throat Hospital

\section{Chi-Yao Hsueh}

Fudan University Eye Ear Nose and Throat Hospital

\section{Liang Zhou ( $\square$ liang.zhou@fdeent.org)}

Fudan University Eye Ear Nose and Throat Hospital https://orcid.org/0000-0002-3647-000X

\section{Primary research}

Keywords: metabolism, prognosis, tissue microarray, laryngeal squamous cell carcinoma, nomogram

Posted Date: November 2nd, 2021

DOI: https://doi.org/10.21203/rs.3.rs-1018287/v1

License: (9) This work is licensed under a Creative Commons Attribution 4.0 International License. Read Full License 


\section{Abstract \\ Background}

A growing body of evidence has suggested the involvement of metabolism in the occurrence and development of tumors. But the link between metabolism and laryngeal squamous cell carcinoma (LSCC) has rarely been reported. This study seeks to understand and explain the role of metabolic biomarkers in predicting the prognosis of LSCC.

\section{Methods}

We identified the differentially expressed metabolism-related genes (MRGs) through RNA-seq data of TCGA (The Cancer Genome Atlas) and GSEA (Gene set enrichment analysis). After the screening of protein-protein interaction (PPI), hub MRGs were analyzed by least absolute shrinkage and selection operator (LASSO) and Cox regression analyses to construct a prognostic signature. Kaplan-Meier survival analysis and the receiver operating characteristic (ROC) was applied to verify the effectiveness of the prognostic signature in four cohorts (TCGA cohort, GSE27020 cohort, TCGA-sub1 cohort and TCGAsub2 cohort). The expressions of the hub MRGs in cell lines and clinical samples were verified by quantitative reverse transcriptase PCR (qRT-PCR). The immunofluorescence staining of the tissue microarray (TMA) was carried out to further verify the reliability and validity of the prognostic signature. Cox regression analysis was then used to screen for independent prognostic factors of LSCC and a nomogram was constructed based on the results.

\section{Results}

Among the 180 differentially expressed MRGs, 14 prognostic MRGs were identified. A prognostic signature based on two MRGs (GPT and SMS) was then constructed and verified via internal and external validation cohorts. Compared to the adjacent normal tissues, SMS expression was higher while GPT expression was lower in LSCC tissues, indicating poorer outcomes. The risk score proved the prognostic signature as an independent risk factor for LSCC in both internal and external validation cohorts. A nomogram based on these results was developed for clinical application.

\section{Conclusions}

Differentially expressed MRGs were found and proven to be related to the prognosis of LSCC. We constructed a novel prognostic signature based on MRGs in LSCC for the first time and verified via different cohorts from both databases and clinical samples. A nomogram based on this prognostic signature was developed. 


\section{Background}

Laryngeal squamous cell carcinoma (LSCC) is a most common cancer of the upper respiratory tract. Smoking and drinking are well recognized as risk factors for LSCC[1]. The Global Cancer Observatory reported more than 170,000 new cases of laryngeal cancer and more than 94,000 deaths due to laryngeal cancer in 2018[2]. Despite the continuous improvement in the treatment and management, the prognosis for LSCC patients remains unsatisfactory and the mortality rate is still high[3]. Most LSCC cases are in the locoregionally advanced stage at the time of diagnosis[4]. The recurrence rate is high and the patients often develop resistance to chemotherapy or radiotherapy. The clinical outcome for patients with advanced laryngeal cancer remains poor[5]. Hence, identifying a clinically tractable biomarker that can effectively predict the prognosis of LSCC would greatly benefit both the clinicians and the patients.

Metabolism, a series of reactions within cells of living organisms to sustain life, involves many interconnected cellular pathways to ultimately provide cells with the energy to function[6]. The research on metabolism aims to explore and solve biological problems from the level of molecular characters and results [6]. Recent studies have shown that metabolic reprogramming regulates oncogenesis and tumor development. Gong et al found that lipid metabolism reprogramming in cancer-associated fibroblasts potentiates migration of colorectal cancer cells[7]. In breast tumors, ADHFE1 and MYC signaling contributes to the accumulation of $\mathrm{D}-2 \mathrm{HG}$, an oncogenic metabolite and potential driver of disease progression[8]. Tobacco smoking is shown to induce metabolic reprogramming in renal cell carcinoma[9]. These reprogramming activities can help to meet the bioenergy, biosynthesis, and redox needs of cancer cells, and thus are now considered as markers of cancer[10]. Although metabolism in cancer has attracted more and more attention, little research has been done regarding the relationship between LSCC and metabolic markers.

In this study, we analyzed metabolomic profiling of LSCC patients and classified them into different groups. Through the mutual verification of different databases and tissue microarray (TMA), we developed and verified a novel prognostic signature based on metabolism-related genes (MRGs), which might eventually provide better LSCC patient classification and guide treatment from the perspective of metabolism.

\section{Materials And Methods}

\section{Data acquisition}

We collected RNA-seq data and microarray data to prove the effectiveness of this study. The RNA-seq data were originated from 111 LSCC tumor samples and 12 matched normal tissues in The Cancer Genome Atlas (TCGA, https://portal.gdc.cancer.gov)[11]. The clinical information of 111 LSCC patients from TCGA would be demonstrated in Supplementary Table 1. Also, we filtered the microarray data and download GSE27020 dataset from Gene Expression Omnibus (GEO, https://www.ncbi.nlm.nih.gov/geo/) 
[12] which including 75 non-recurrent and 34 recurrent LSCC patients for subsequent verification of the prognosis signature[13].

\section{Identification of differentially expressed MRGs}

We collected all the metabolic-related pathways and corresponding genes contained in the Gene set enrichment analysis (GSEA) database (Supplementary Table 2)[14]. We extracted the expression of metabolic-related genes from TCGA and conducted differential expression analysis by "limma"[15] package in R software (version: x64 3.6.1). We set the Fold change (FC) equal to the expression of genes in LSCC tissues divided by the expression in normal tissues. The screening condition were $|\log (\mathrm{FC})| \geq 1$ and an adjusted $p$-value $<.01$.

\section{Pathway enrichment analysis and protein-protein interaction (PPI) analysis}

In order to explore the biological functions of differentially expressed MRGs, we performed Gene Ontology (GO)[16] and KEGG (Kyoto Encyclopedia of Genes and Genomes) analysis[17]. Then, we introduced these genes into String database (https://string-db.org/)[18] to eliminated the genes with poor connectivity and visualized the result through Cytoscape software (Version 3.7.1)[19].

\section{Construction and validation of a prognosis signature based on MRGs}

90 patients with LSCC were enrolled in TCGA cohort after integrating the clinical information. Univariate Cox regression analysis was performed to screen out prognostic MRGs. Least absolute shrinkage and selection operator (LASSO) analysis and multivariate Cox regression analysis were applied to construct a prognosis signature. The risk score formula of the prognosis signature was as follows: Risk score $=$ coef * $\operatorname{Exp}($ geneA $)+$ coef * $\operatorname{Exp}($ geneB $)+$ coefi * Expi (genei) $[20,21] .90$ patients were divided into high and low-risk group according to the median risk score (1.244). Kaplan-Meier survival analysis and receiver operating characteristic (ROC) curve were calculated to measure the reliability and validity of the signature as internal validation. The same analysis method was applied in GSE27020 cohort as external validation to verify the effectiveness of the prognosis signature we developed based on RBPs in predicting disease-free survival (DFS) of LSCC. Moreover, in order to further verify the universality of the prognosis signature, 90 patients in TCGA cohort were randomly divided into TCGA-sub1 and TCGA-sub2 cohort according to the ratio of 1:1, the same analysis method was applied.

\section{Ethics statement and tissue specimens}

After being approved by the Ethical Committees of Eye and ENT Hospital, Fudan University, a total of 62 paired samples from LSCC patients were collected between September 2020 to July 2021. LSCC and adjacent normal tissues, which were $\geq 3 \mathrm{~cm}$ distal to the incisal edge, were stored at $-80^{\circ} \mathrm{C}$ and used for quantitative reverse transcriptase PCR (qRT-PCR). All patients were agreed and informed consent was obtained for all LSCC patients. 


\section{Cell culture}

The LSCC cell lines LSCC-31, FD-LSC-1, AMC-HN8 and Tu686 from LSCC patients were prepared and tested. LSCC-31 and FD-LSC-1 was cultured in BEGM (CC-3170 Lonza), Tu686 was cultured in DMEM (Gibco), while AMC-HN8 in RPMI-1640 (Gibco), with 10\% fetal bovine serum (Gibco) at $37^{\circ} \mathrm{C}$ in the presence of $5 \% \mathrm{CO} 2$. HuLa-PC, a cell line derived from posterior commissure of the larynx, was obtained from ATCC (Gaithersburg, Maryland) and cultured in Dermal Cell Basal Medium (ATCC $\circledast$ PCS-200030TM).

\section{qRT-PCR}

Total RNA was isolated from tissues and cell lines with TRIzol reagent (Invitrogen, Thermo Fisher Scientific), and then reverse transcribed using Evo M-MLV RT Kit with gDNA Clean for qPCR (AG11711). qRT-PCR was conducted using SYBR Green Premix Pro Taq HS qPCR Kit (AG11701) for mRNA with ABI 7500 Real-Time PCR System (Life Technologies, Shanghai, China), and the housekeeping gene GAPDH was used as an internal control. The primers were synthesized by Sangon Biotech (Shanghai) Co., Ltd. The sequences of all primers were listed in Supplementary Table 3.

\section{Immunofluorescence of tissue microarrays (TMA)}

The tissue microarrays, which including 72 paired patients with head and neck squamous cell carcinoma (HNSCC), was obtained from Eye and ENT Hospital, Fudan University (FDEENT). After filtering out patients with hypopharyngeal carcinoma and fragments, 51 patients with LSCC remained. Immunofluorescence staining was performed on 51 paired samples to detect GPT (ab236658, ABCAM, 1:100 dilution) $\square S M S$ (ab247063, ABCAM, 1:3000 dilution) and DAPI (G1012, Servicebio) expression. TMA was scanned using the microscope slide scanner (Pannoramic MIDI: 3Dhistech) after staining with the appropriate antibody. All scanned cores or slides were individually detected and quantified using ImageJ software[22]. GPT and SMS positive staining were calculated relative to DAPI staining. Integrating survival information, we conducted receiver operating characteristic $(\mathrm{ROC})$ and survival analysis of risk score (GPT+SMS), GPT and SMS in LSCC tissues. ROC analysis was performed to identify the sensitivity and specificity of risk score and the optimal cutoff value for predicting overall survival (OS). According to the cutoff value, patients were divided into two groups. The prognostic difference between the two groups was then analyzed using a Kaplan-Meier estimator with a log rank test.

\section{Independent prognosis analysis}

In order to further evaluate the predictive ability of the prognosis signature, we used Cox regression analysis to analyze whether the prognosis signature could act as an independent prognostic factor for LSCC both in TCGA cohort and FDEENT cohort.

\section{Building a nomogram based on the prognosis signature}

Based on results of independent prognosis analysis, we tried to build a nomogram by "rms" package to facilitate the application of the prognosis signature. Calibration curves and ROC curves were used to 
verify the validity of the signature.

\section{Statistical analysis}

All statistical analyses were carried out by using R software (version: x64 3.6.1) and GraphPad Prism 7 software. A $p$-value $<.05$ was regarded as statistically significant.

\section{Results}

\section{Identification of differentially expressed MRGs}

The flow chart of this study was presented in Figure 1. We obtained the RNA-seq data of MRGs from TCGA and GSEA, and 180 differentially expressed MRGs were screened out by filter conditions (Supplementary Table 3). The differentially expressed MRGs were visualized in volcano plot (Figure 2A) and the top 80 MRGs with significant adjusted $p$-value were shown in a heatmap (Figure 2B).

\section{Pathway enrichment analysis and PPI analysis}

The results of GO analysis showed that the differentially expressed MRGs were mainly enriched in small molecule catabolic process, carboxylic acid biosynthetic process, organic acid biosynthetic process, cellular amino acid metabolic process, alpha-amino acid metabolic process, alcohol metabolic process, organic acid catabolic process, carboxylic acid catabolic process, coenzyme binding, and fatty acid metabolic process (Figure 2C). KEGG analysis demonstrated that the differentially expressed MRGs were significantly accumulated in Chemical carcinogenesis, Drug metabolism - cytochrome P450, Drug metabolism - other enzymes, Metabolism of xenobiotics by cytochrome P450, Purine metabolism, Retinol metabolism, Biosynthesis of amino acids, Biosynthesis of cofactors, Glutathione metabolism, Glycolysis / Gluconeogenesis (Figure 2D). At the same time, 173 MRGs with high connectivity were used to establish a PPI network (Figure 3A-B). Three most important sub-networks were screened out (Figure 3C-F) and the enrichment results of sub-networks were presented in Supplementary Table 4.

\section{Construction and validation of a prognosis signature based on MRGs}

In TCGA cohort, 14 prognostic genes were identified via univariate Cox regression analysis (Table 1). Subsequently, an MRGs-related prognostic signature was constructed by LASSO analysis (Figure 4) and multivariate Cox regression analysis (Figure 4C, Table 2). The risk score of the prognostic signature could be calculated as follows: (Exp GPT * -1.922494852) + (Exp SMS * 0.772558166).

To evaluate the predictive effectiveness of the prognostic signature, we divided the patients of the TCGA cohort $(n=90)$ into the high- and low-risk groups according to the median risk score. The overall survival (OS) of the high-risk group was poorer than that of the low-risk group (Figure $5 \mathrm{~A}$ ). The area under curves (AUC) of TCGA cohort was 0.748 (Figure $5 B$ ). The survival status, risk score and the hub MRGs expression of the two groups were displayed in Figure 5C-E. 
In the external validation (GSE27020 cohort, $n=109$ ), the DFS of the high-risk group was poorer than that of the low-risk group (Supplementary Figure 1A-B). The DFS status, risk score and the hub MRGs expression of the two groups were displayed in Supplementary Figure 1C-E. The results suggested that the prognostic signature we developed was not only effective for stratification of laryngeal cancer survival, but also effective for recurrence patients.

In the internal validation (TCGA-sub1 cohort, $n=45$; TCGA-sub2 cohort, $n=45$ ), the OS of the high-risk group was poorer than that of the low-risk group in both cohorts (Supplementary Figure 2-3). The AUCs of both cohorts were shown in Supplementary Figure 2-3, respectively.

\section{Higher SMS expression and lower GPT expression in LSCC tissues indicating a poorer outcome}

In order to verify the expressions of GPT and SMS, 62 LSCC tissues with their paired adjacent normal tissues and five cell lines were selected and tested by qRT-PCR. SMS in LSCC tissues was up-regulated compared to the adjacent normal tissues, while GPT was the opposite (Figure 6A-B), which coincided with our findings from TCGA database (Figure 6E-F). Consistent results were also observed in cell lines (Figure $6 C-D)$.

In TMA (FDEENT cohort, $\mathrm{n}=51$ ), we noted that GPT protein (in pink) was localized primarily in the epithelium (Figures 7A-F) and down-regulated in LSCC tissues compared to adjacent normal tissues (Figures 7G). ROC curve showed that the AUC was $0.7963(p=0.0005)$ and a value of 0.355 was the best balance between the sensitivity and specificity for predicting OS (Figure $7 \mathrm{H})$. Survival analysis showed that the low expression of GPT was related to a lower OS rate among LSCC patients $(p=0.0287)$ (Figure $7 \mathrm{7l)}$. SMS protein was analyzed in the same way and the results were displayed in Supplementary Figure 4.

To validate our findings, we integrated GPT and SMS expression according to the prognosis signature (GPT * - 1.922494852 + SMS * 0.772558166). In FDEENT cohort $(n=51)$, we found that the AUC of risk score (GPT+SMS) was $0.798(p=0.0005)$ (Figure $7 \mathrm{~J})$ and the OS of the high-risk group was poorer than that of the low-risk group ( $p=0.0387$ ) (Figure 7K). These results were also consistent with our findings.

\section{Independent prognosis analysis}

The FDEENT cohort and TCGA cohort were assessed by independent prognosis analysis. The results showed that the risk score and tumor length were significant independent risk factors in FDEENT cohort (Figure 8A-B), while the risk score, gender and stage $\mathrm{N}$ were significant independent risk factors in TCGA cohort (Figure 8C-D).

\section{Building a nomogram based on the prognosis signature}

Based on TCGA cohort, we built a nomogram (Figure 8E) to make the prognosis signature more convenient for clinical application. Based on the score of subitems (including the risk score and other significant independent risk factors), the 1 -, 3 -, and 5-year OS of patients with LSCC could be predicted. 
Calibration curves indicated that the predicted value of the risk score was closer to the actual observation value with the increase in number of years (Figure 8F-H). At the same time, we found that the AUC of the risk score was higher than that of the other significant independent risk factors in 1 -, 3 -, and 5-year OS (Figure 8J-L).

\section{Discussion}

LSCC, which is of epithelial origin, is a most common tumor of the upper respiratory tract[23]. Poor living habits, such as imbalanced diet, smoking, and alcohol consumption, are the main risk factors contributing to laryngeal cancer[24]. As an important feature of tumors, metabolic abnormality could adjust the microenvironment to meet the requirement of the constantly growing tumor cells[25]. Recently, more and more studies have focused on tumor metabolism to elucidate the pathogenesis of LSCC. Hu reported that PCK2 down-regulation inhibited the invasion, migration, and proliferation of laryngeal cancer under hypoxia, and therefore could be used as a new strategy for laryngeal cancer therapy[26]. Using liquid chromatograph-mass spectrometry (LC-MS) technology and molecular biology experiments, Zhao et al. found that fatty acid desaturase 1 (FADS1) promoted the progression of LSCC through activating $\mathrm{AKT} / \mathrm{mTOR}$ signaling pathway[27].

In this study, we utilized several public databases and screened out prognostic metabolic markers of LSCC by bioinformatics analysis. It was worth noting that the results of GO and KEGG pathway enrichment analyses were all related to metabolism, such as purine metabolism, glycometabolism, lipid metabolism, and pyrimidine metabolism. Prognostic MRGs were screened out and a novel prognostic signature was then constructed based on the two MRGs (GPT and SMS).

GPT (glutamic pyruvic transaminase), also known as alanine aminotransferase, is strongly involved in cellular metabolism and cancer cell turnover[28]. GPT catalyzes the transfer of amino groups to generate products in gluconeogenesis and amino acid metabolism[29]. Accumulating evidence has shown that the expression level of GPT may be related to the prognosis of various cancers, including bladder cancer[30], pancreatic cancer[31], urothelial carcinoma[32], and small cell lung cancer[33]. The abnormal expression of GPT has a variety of causes, and long-term drinking can increase GPT expression[34]. Alcohol consumption is also an accepted risk factor for laryngeal cancer. Therefore, we speculated that chronic alcohol abuse might lead to the abnormal expression of GPT, thereby promoting the occurrence and development of laryngeal cancer. GPT is a common index to evaluate liver function, and the detection method is simple and cheap. Here, we found for the first time that GPT could be used as an important predictor of LSCC. If the role of GPT can be further proved by cohort and molecular biology experiments, it will bring great convenience for the diagnosis and prognosis of laryngeal cancer.

SMS (spermine synthase) is often associated with Snyder-Robinson syndrome (SRS), because SRS is caused by alterations in the human SMS gene[35,36]. The level of polyamines, the aliphatic cations present in all cells, is intricately controlled by biosynthetic and catabolic enzymes. SMS, a highly specific aminopropyltransferase[37], is a biosynthetic enzyme[38]. Previous studies have shown that the 
increased activity of polyamine-producing enzymes leads to the up-regulation of polyamine level, which maintains the growth and proliferation of tumor cells[39]. Guo[40]found that SMS was overexpressed in colorectal cancer and maintained the colorectal cancer cell survival. Also, the high expression of SMS mRNA was related to a low overall survival rate and metastasis of triple-negative breast cancer[41]. As a spermine metabolism-related gene, the role of SMS in LSCC has not been reported, and its specific molecular mechanism is not clear. In our study, SMS expression was up-regulated in LSCC tissues and related to the prognosis of LSCC patients. We also found SMS could be a prognostic signature to predict the survival of LSCC patients. These results may shed light on the relationship between spermine metabolism and LSCC.

Concordant with previous reports, SMS was up-regulated while GPT was down-regulated in LSCC tissues compared to adjacent normal tissues as verified by clinical samples and multiple datasets in our study. Based on the expressions of SMS and GPT, LSCC patients were divided into the high and low risk groups. Five different cohorts, including internal and external validation cohorts, were analyzed and tested to verify the accuracy and reliability of the prognosis signature. The risk score of the signature was the best independent prognostic factor in both TCGA and FDEENT cohorts. Therefore, a nomogram based on the risk score was developed, which might contribute to treatment decision making.

In the previous studies, Chen[42] and Li[43] developed metabolism-related signatures to predict the prognosis of head and neck squamous cell carcinoma, while Hu[44] identified a combined lipid metabolism-related signature for oral squamous cell carcinoma (OSCC). To our knowledge, our study is the first to develop and validate an MRG signature for laryngeal squamous cell carcinoma (LSCC). This signature showed a higher AUC value than the other studies based on TCGA cohort (Figure 8I), indicating a higher sensitivity and specificity. In addition, compared with other prognostic signatures composed of multiple genes, this signature derived from two MRGs is easier to apply in clinical practice.

Also, there are some deficiencies in our research. First, only five cohorts were incorporated to verify this prognostic signature. In future study, more cohorts and LSCC patients should be included to reduce the deviation of racial and geographic distribution. Second, our findings were based on retrospective studies, prospective clinical trials should be conducted. More importantly, in vivo and in vitro experiments on GPT and SMS are needed.

\section{Conclusion}

In summary, our study identified differentially expressed MRGs and constructed a novel prognostic signature derived from MRGs in LSCC for the first time. Being able to distinguish LSCC patients with different risk scores, this novel signature could make precise prognosis of LSCC. A nomogram based on this prognostic signature was also developed.

\section{Abbreviations}


LSCC: laryngeal squamous cell carcinoma; MRGs: metabolism-related genes; TCGA: The Cancer Genome Atlas; GSEA: Gene set enrichment analysis; PPI: protein-protein interaction; LASSO: least absolute shrinkage and selection operator; ROC: receiver operating characteristic; qRT-PCR: quantitative reverse transcriptase PCR; TMA: tissue microarray; GEO: Gene Expression Omnibus; Gene Expression Omnibus; FC: Fold change; GO: Gene Ontology; KEGG: Kyoto Encyclopedia of Genes and Genomes; DFS: diseasefree survival; HNSCC: head and neck squamous cell carcinoma; FDEENT: Eye and ENT Hospital, Fudan University; OS: overall survival; FADS1: fatty acid desaturase 1; GPT: glutamic pyruvic transaminase; SMS: spermine synthase; SRS: Snyder-Robinson syndrome; OSCC: oral squamous cell carcinoma.

\section{Declarations}

\section{Acknowledgements}

Not applicable.

\section{Authors' contributions}

YS and QH performed the data curation and analysis. YZ analyzed and interpreted the results. LZ and HC drafted and reviewed the manuscript. All authors read and approved the final manuscript. YS, QH and YZ contributed equally to this study.

\section{Funding}

The present study was supported by grants from the National Natural Science Foundation of China [No. 81972529] and the Science and Technology Commission of Shanghai Municipality [No. 19411961300].

\section{Availability of data and materials}

The datasets analyzed during the current study are available from the cor-responding author Liang Zhou (zhoulent@126.com) on reasonable request. The data that support the findings of this study are available at the TCGA data portal (https://tcga-data.nci.nih.gov/tcga/) and the comprehensive Gene Expression Omnibus (GEO;https://www.ncbi.nlm.nih.gov/geo/).

\section{Ethics approval and consent to participate}

All the subjects involved have been informed of the purpose and significance of this study, signed the informed consent and provided the necessary biological samples for testing and analysis. The study has been approved by the The Ethical Committees of Eye and ENT Hospital, Fudan University.

\section{Consent for publication}

All the subjects involved have agreed to publish relevant research results.

\section{Competing interests}


The authors declare that they have no competing interests.

\section{References}

1. Choong N, Vokes E. Expanding role of the medical oncologist in the management of head and neck cancer. CA Cancer J Clin. 2008;58:32-53.

2. Bray F, Ferlay J, Soerjomataram I, Siegel RL, Torre LA, Jemal A. Global cancer statistics 2018 : GLOBOCAN estimates of incidence and mortality worldwide for 36 cancers in 185 countries. CA Cancer J Clin. 2018;68:394-424.

3. Song L, Zhang S, Yu S, Ma F, Wang B, Zhang C, et al. Cellular heterogeneity landscape in laryngeal squamous cell carcinoma. Int J Cancer. 2020;147:2879-90.

4. Allegra E, Puzzo L, Zuccalà V, Trapasso S, Vasquez E, Garozzo A, et al. Nuclear BMI-1 expression in laryngeal carcinoma correlates with lymph node pathological status. World J Surg Oncol. 2012;10:206.

5. Greco A, Rizzo MI, De Virgilio A, Gallo A, Fusconi M, Pagliuca G, et al. Cancer stem cells in laryngeal cancer: what we know. Eur Arch Otorhinolaryngol. 2016;273:3487-95.

6. Judge A, Dodd MS. Metabolism. Essays Biochem. 2020;64:607-47.

7. Gong J, Lin Y, Zhang H, Liu C, Cheng Z, Yang X, et al. Reprogramming of lipid metabolism in cancerassociated fibroblasts potentiates migration of colorectal cancer cells. Cell Death Dis. 2020;11:267.

8. Mishra P, Tang W, Putluri V, Dorsey TH, Jin F, Wang F, et al. ADHFE1 is a breast cancer oncogene and induces metabolic reprogramming. J Clin Invest. 2018;128:323-40.

9. Reigle J, Secic D, Biesiada J, Wetzel C, Shamsaei B, Chu J, et al. Tobacco smoking induces metabolic reprogramming of renal cell carcinoma. J Clin Invest. 2021;131:140522.

10. DeBerardinis RJ, Chandel NS. Fundamentals of cancer metabolism. Sci Adv. 2016;2:e1600200.

11. Blum A, Wang P, Zenklusen JC. SnapShot: TCGA-Analyzed Tumors. Cell. 2018;173:530.

12. Barrett T, Wilhite SE, Ledoux P, Evangelista C, Kim IF, Tomashevsky M, et al. NCBI GEO: archive for functional genomics data sets-update. Nucleic Acids Res. 2013;41:D991-995.

13. Fountzilas E, Kotoula V, Angouridakis N, Karasmanis I, Wirtz RM, Eleftheraki AG, et al. Identification and validation of a multigene predictor of recurrence in primary laryngeal cancer. PLoS One. 2013;8:e70429.

14. Subramanian A, Tamayo P, Mootha VK, Mukherjee S, Ebert BL, Gillette MA, et al. Gene set enrichment analysis: a knowledge-based approach for interpreting genome-wide expression profiles. Proc Natl Acad Sci U S A. 2005;102:15545-50.

15. Ritchie ME, Phipson B, Wu D, Hu Y, Law CW, Shi W, et al. limma powers differential expression analyses for RNA-sequencing and microarray studies. Nucleic Acids Res. 2015;43:e47.

16. Gene Ontology Consortium. Gene Ontology Consortium: going forward. Nucleic Acids Res. 2015;43:D1049-1056. 
17. Kanehisa M, Furumichi M, Tanabe M, Sato Y, Morishima K. KEGG: new perspectives on genomes, pathways, diseases and drugs. Nucleic Acids Res. 2017;45:D353-61.

18. von Mering C, Huynen M, Jaeggi D, Schmidt S, Bork P, Snel B. STRING: a database of predicted functional associations between proteins. Nucleic Acids Res. 2003;31:258-61.

19. Shannon P, Markiel A, Ozier O, Baliga NS, Wang JT, Ramage D, et al. Cytoscape: a software environment for integrated models of biomolecular interaction networks. Genome Res. 2003;13:2498-504.

20. Shen C, Liu J, Wang L, Liang Z, Niu H, Wang Y. Identification of metabolism-associated genes and construction of a prognostic signature in bladder cancer. Cancer Cell Int. 2020;20:538.

21. Wang Z, Embaye KS, Yang Q, Qin L, Zhang C, Liu L, et al. Establishment and validation of a prognostic signature for lung adenocarcinoma based on metabolism-related genes. Cancer Cell Int. 2021;21:219.

22. Schneider CA, Rasband WS, Eliceiri KW. NIH Image to ImageJ: 25 years of image analysis. Nat Methods. 2012;9:671-5.

23. He G, Yao W, Li L, Wu Y, Feng G, Chen L. LOXL1-AS1 contributes to the proliferation and migration of laryngocarcinoma cells through miR-589-5p/TRAF6 axis. Cancer Cell Int. 2020;20:504.

24. Garavello W, Lucenteforte E, Bosetti C, Talamini R, Levi F, Tavani A, et al. Diet diversity and the risk of laryngeal cancer: a case-control study from Italy and Switzerland. Oral Oncol. 2009;45:85-9.

25. Reina-Campos M, Moscat J, Diaz-Meco M. Metabolism shapes the tumor microenvironment. Curr Opin Cell Biol. 2017;48:47-53.

26. Hu Y, Deng K, Pan M, Liu S, Li W, Huang J, et al. Down-regulation of PCK2 inhibits the invasion and metastasis of laryngeal carcinoma cells. Am J Transl Res. 2020;12:3842-57.

27. Zhao R, Tian L, Zhao B, Sun Y, Cao J, Chen K, et al. FADS1 promotes the progression of laryngeal squamous cell carcinoma through activating AKT/mTOR signaling. Cell Death Dis. 2020;11:272.

28. Bezan A, Mrsic E, Krieger D, Stojakovic T, Pummer K, Zigeuner R, et al. The Preoperative AST/ALT (De Ritis) Ratio Represents a Poor Prognostic Factor in a Cohort of Patients with Nonmetastatic Renal Cell Carcinoma. J Urol. 2015;194:30-5.

29. Wroblewski F, Ladue JS. Serum glutamic pyruvic transaminase in cardiac with hepatic disease. Proc Soc Exp Biol Med. 1956;91:569-71.

30. Ha Y-S, Kim SW, Chun SY, Chung J-W, Choi SH, Lee JN, et al. Association between De Ritis ratio (aspartate aminotransferase/alanine aminotransferase) and oncological outcomes in bladder cancer patients after radical cystectomy. BMC Urol. 2019;19:10.

31. Stocken DD, Hassan AB, Altman DG, Billingham LJ, Bramhall SR, Johnson PJ, et al. Modelling prognostic factors in advanced pancreatic cancer. Br J Cancer. 2008;99:883-93.

32. Nishikawa M, Miyake H, Fujisawa M. De Ritis (aspartate transaminase/alanine transaminase) ratio as a significant predictor of recurrence-free survival in patients with upper urinary tract urothelial carcinoma following nephroureterectomy. Urol Oncol. 2016;34:417.e9-417.e15. 
33. Rawson NS, Peto J. An overview of prognostic factors in small cell lung cancer. A report from the Subcommittee for the Management of Lung Cancer of the United Kingdom Coordinating Committee on Cancer Research. Br J Cancer. 1990;61:597-604.

34. Zambon P, Talamini R, La Vecchia C, Dal Maso L, Negri E, Tognazzo S, et al. Smoking, type of alcoholic beverage and squamous-cell oesophageal cancer in northern Italy. Int $\mathrm{J}$ Cancer. 2000;86:144-9.

35. Cason AL, Ikeguchi Y, Skinner C, Wood TC, Holden KR, Lubs HA, et al. X-linked spermine synthase gene (SMS) defect: the first polyamine deficiency syndrome. Eur J Hum Genet. 2003;11:937-44.

36. Becerra-Solano LE, Butler J, Castañeda-Cisneros G, McCloskey DE, Wang X, Pegg AE, et al. A missense mutation, p.V132G, in the X-linked spermine synthase gene (SMS) causes SnyderRobinson syndrome. Am J Med Genet A. 2009;149A:328-35.

37. Pegg AE, Michael AJ. Spermine synthase. Cell Mol Life Sci. 2010;67:113-21.

38. Thomas T, Thomas TJ. Polyamine metabolism and cancer. J Cell Mol Med. 2003;7:113-26.

39. Arruabarrena-Aristorena A, Zabala-Letona A, Carracedo A. Oil for the cancer engine: The cross-talk between oncogenic signaling and polyamine metabolism. Sci Adv. 2018;4:eaar2606.

40. Guo Y, Ye Q, Deng P, Cao Y, He D, Zhou Z, et al. Spermine synthase and MYC cooperate to maintain colorectal cancer cell survival by repressing Bim expression. Nat Commun. 2020;11:3243.

41. Fahrmann JF, Vykoukal J, Fleury A, Tripathi S, Dennison JB, Murage E, et al. Association Between Plasma Diacetylspermine and Tumor Spermine Synthase With Outcome in Triple-Negative Breast Cancer. J Natl Cancer Inst. 2020;112:607-16.

42. Chen L, He X, Yi S, Liu G, Liu Y, Ling Y. Six Glycolysis-Related Genes as Prognostic Risk Markers Can Predict the Prognosis of Patients with Head and Neck Squamous Cell Carcinoma. Biomed Res Int. 2021;2021:8824195.

43. Li Y, Weng Y, Pan Y, Huang Z, Chen X, Hong W, et al. A Novel Prognostic Signature Based on Metabolism-Related Genes to Predict Survival and Guide Personalized Treatment for Head and Neck Squamous Carcinoma. Front Oncol. 2021;11:685026.

44. Hu Q, Peng J, Chen X, Li H, Song M, Cheng B, et al. Obesity and genes related to lipid metabolism predict poor survival in oral squamous cell carcinoma. Oral Oncol. 2019;89:14-22.

\section{Tables}

Due to technical limitations, tables are only available as a download in the Supplemental Files section.

\section{Figures}



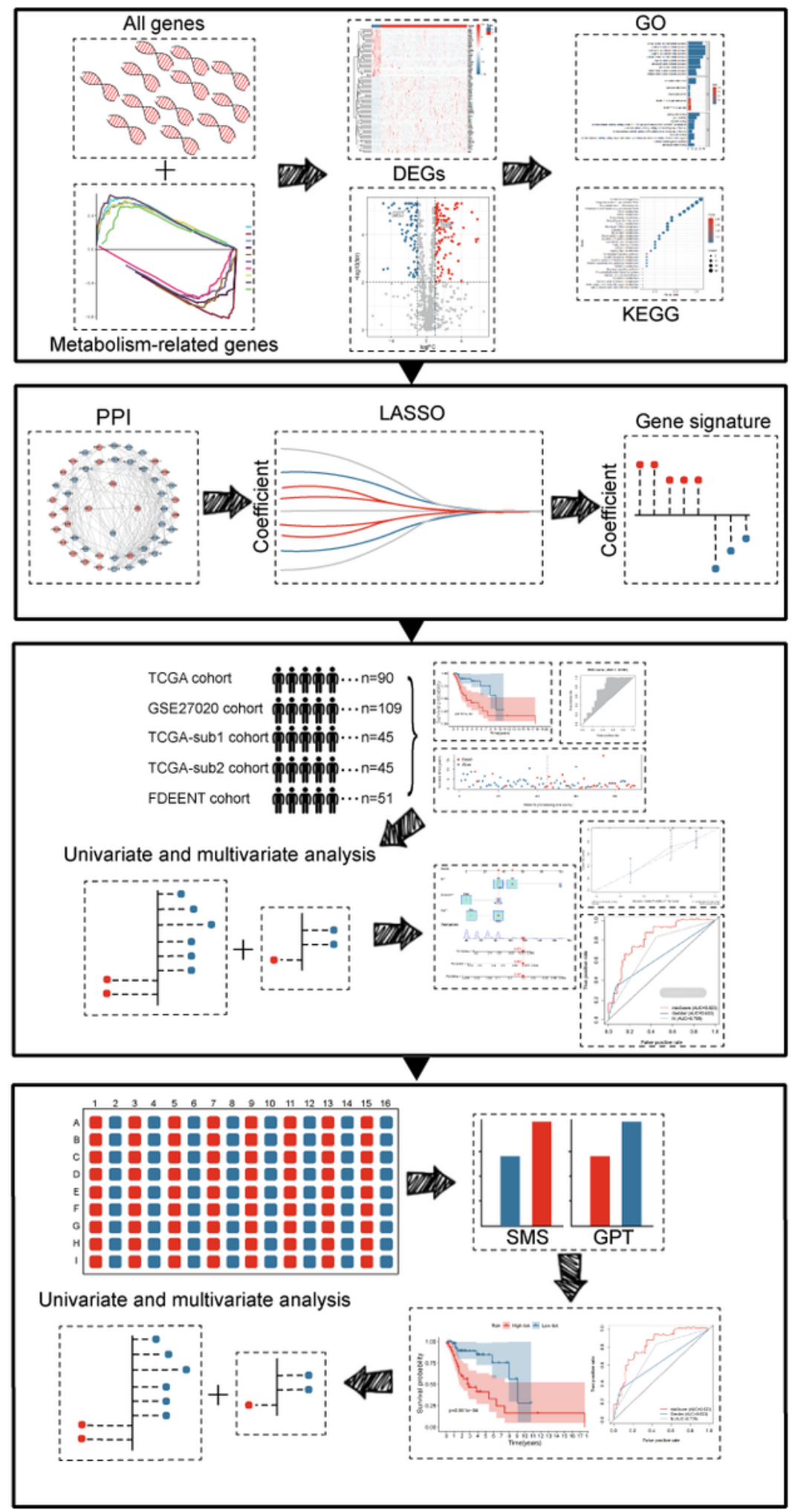

\section{Figure 1}

The flow chart of this study. 
A

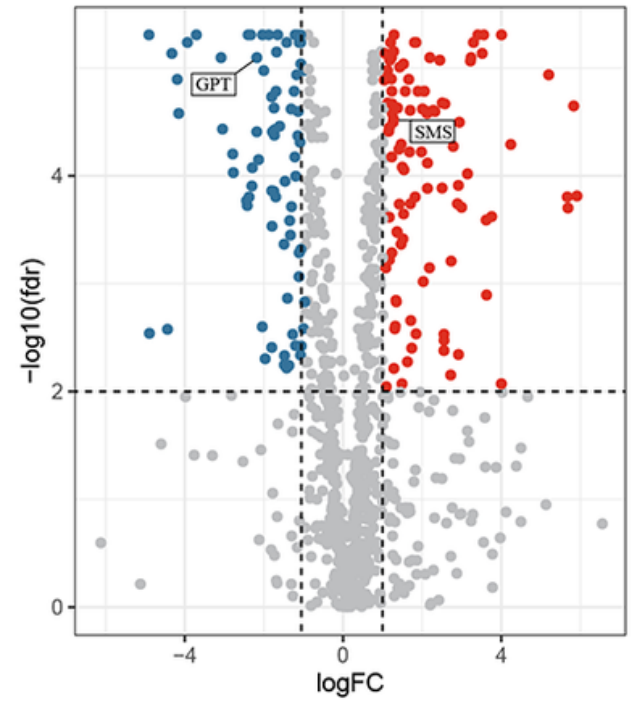

B

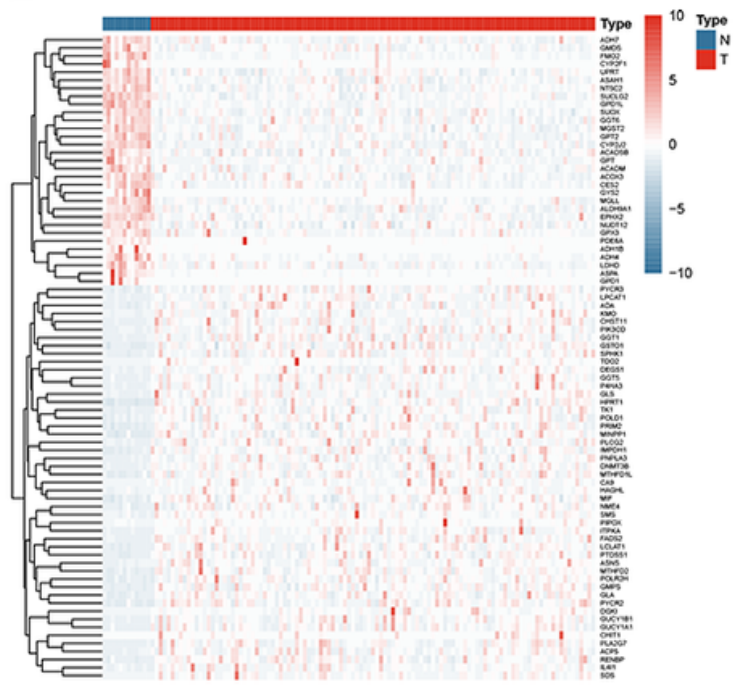

$\mathrm{C}$

Significant

- Down-regulated(76)

- Unchanged

- Up-regulated(104)

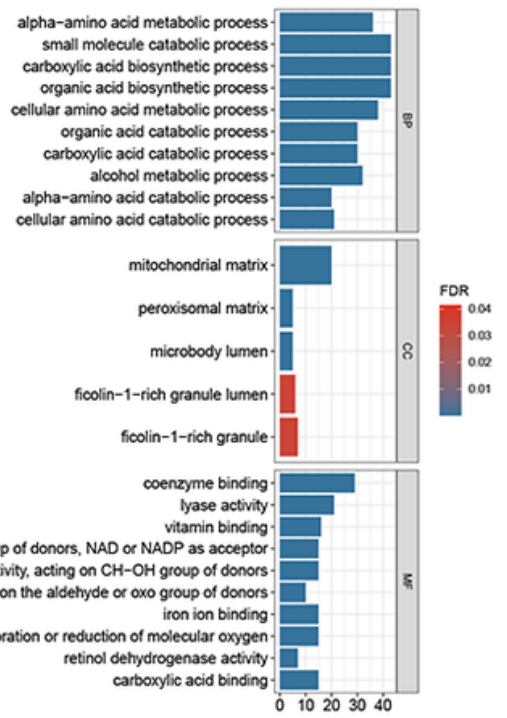

$\mathrm{D}$

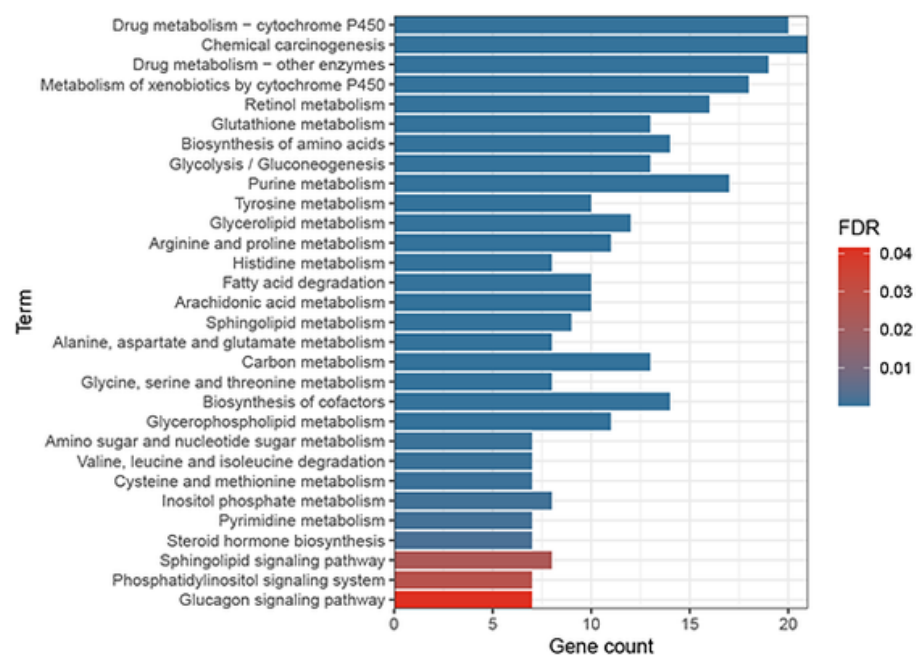

Figure 2

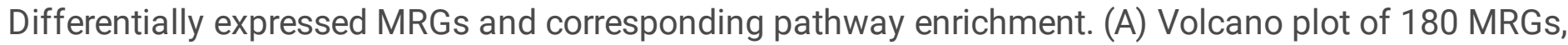
red indicates up-regulated MRGs and blue indicates down-regulated MRGs. (B) Heatmap of the top 80 MRGs with significant adjusted p-value. (C) The GO terms with significant difference. (D) The KEGG pathways with significant difference. 
A

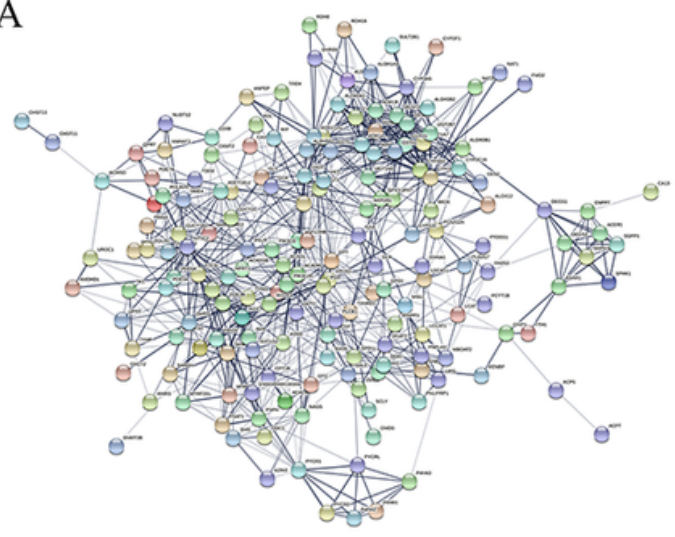

D

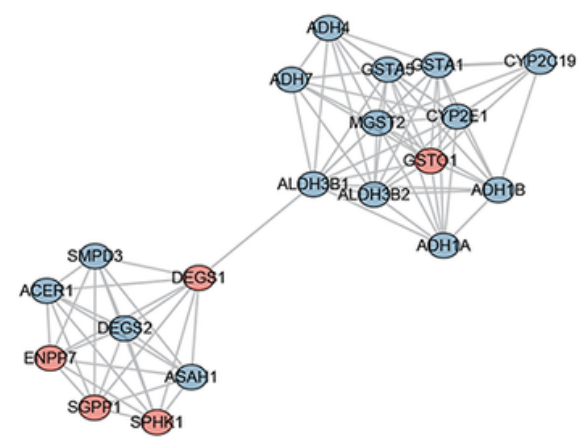

B

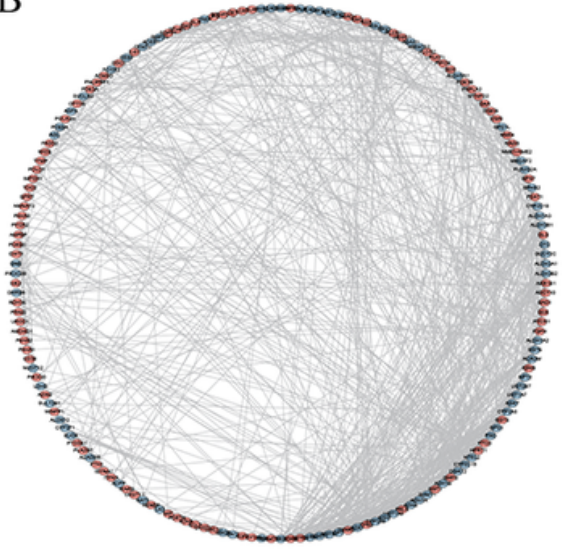

E

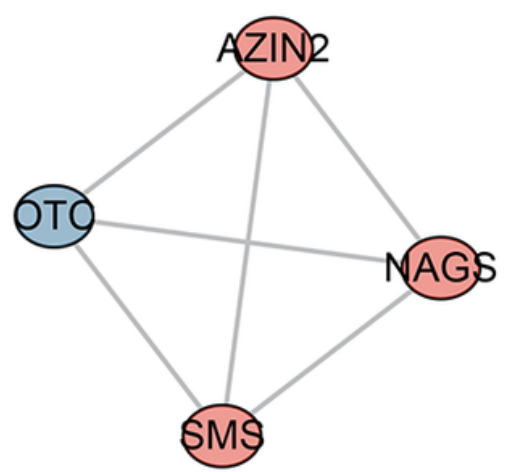

C

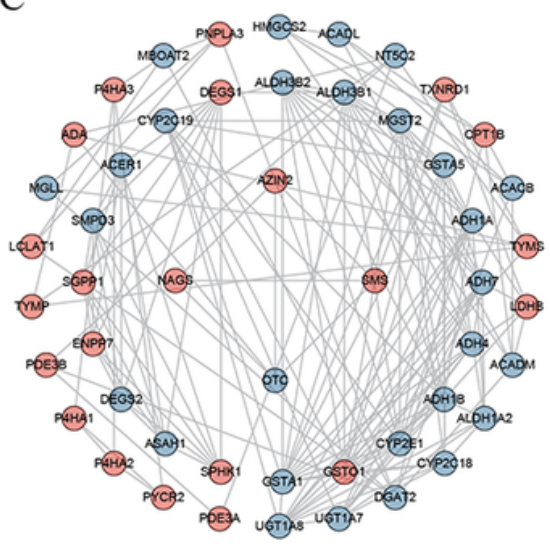

F

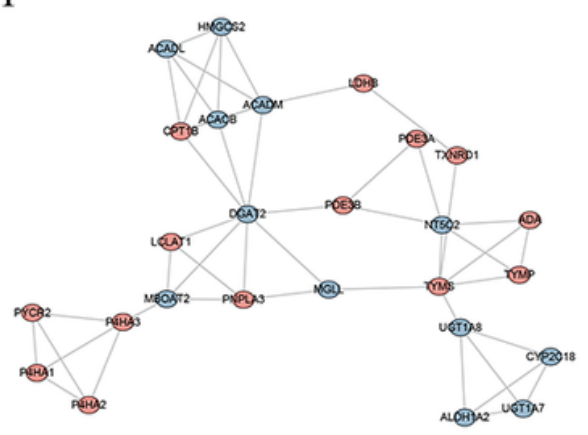

\section{Figure 3}

PPI analysis of different expressed MRGs. (A, B) Visualization of the PPI network, red indicates upregulated MRGs and blue indicates down-regulated MRGs. (C, D, E, F) The three most important subnetworks. 
A

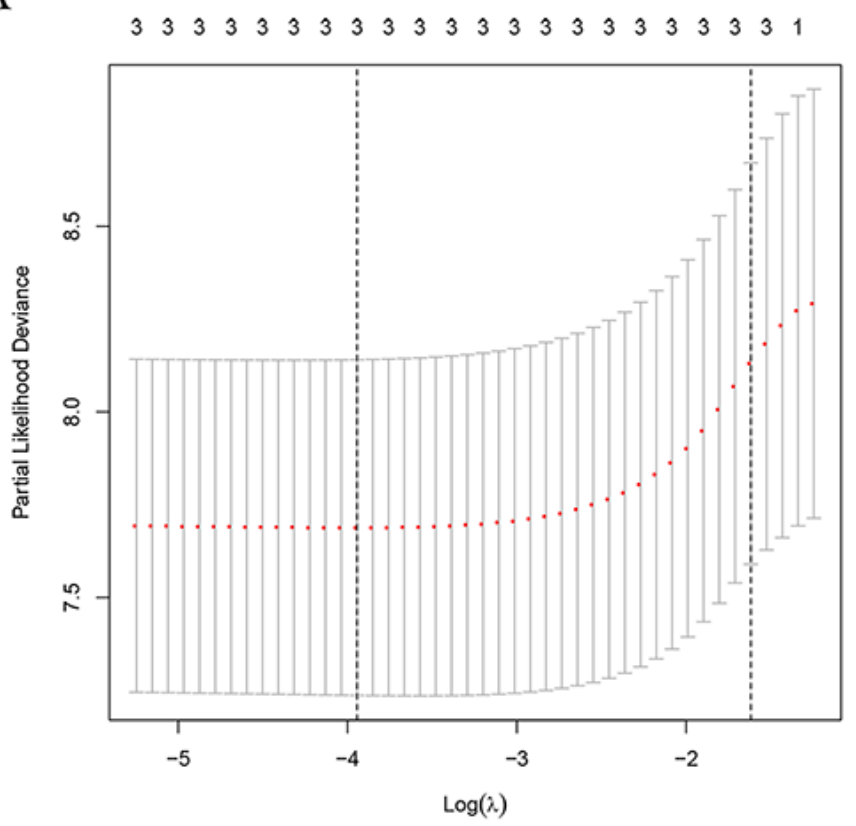

B

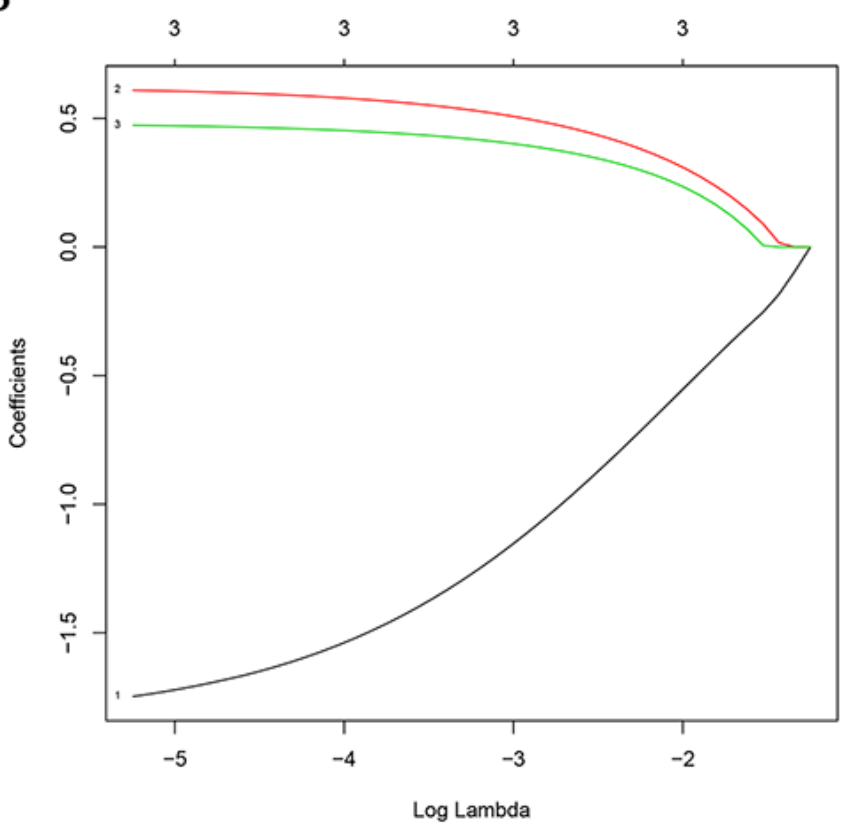

$\mathrm{C}$

Hazard ratio

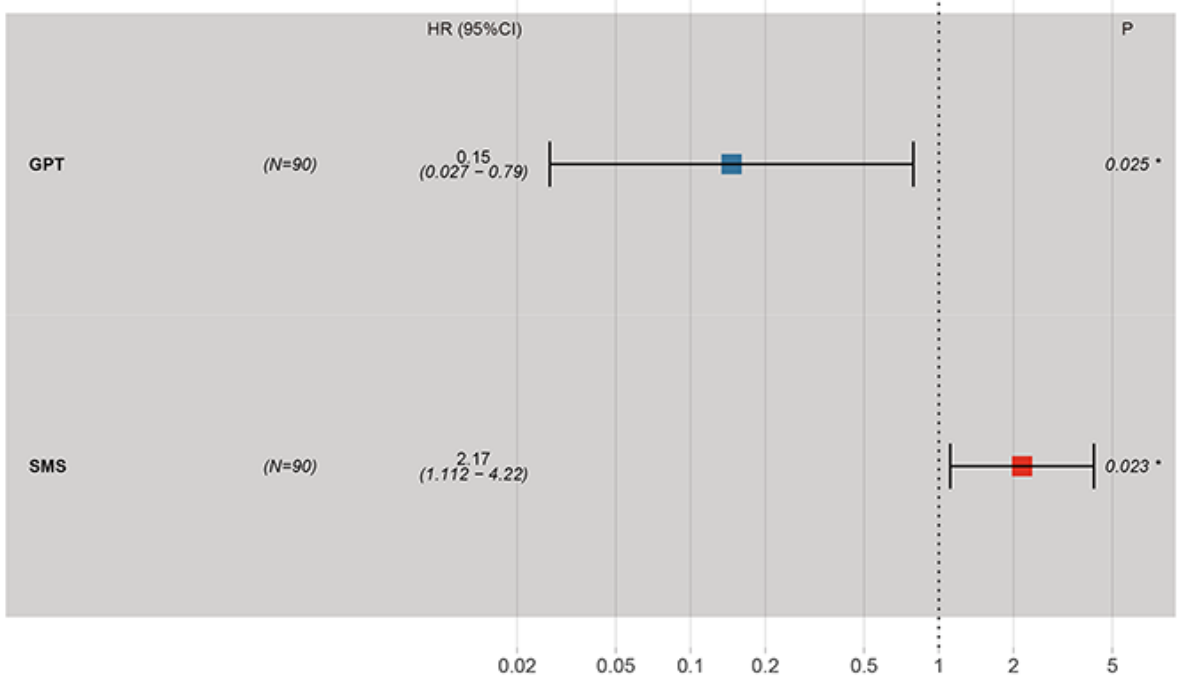

Figure 4

The building process of the prognostic signature. (A) LASSO deviance profiles. (B) LASSO coefficient profiles. (C) Multivariate Cox regression analysis. 
A

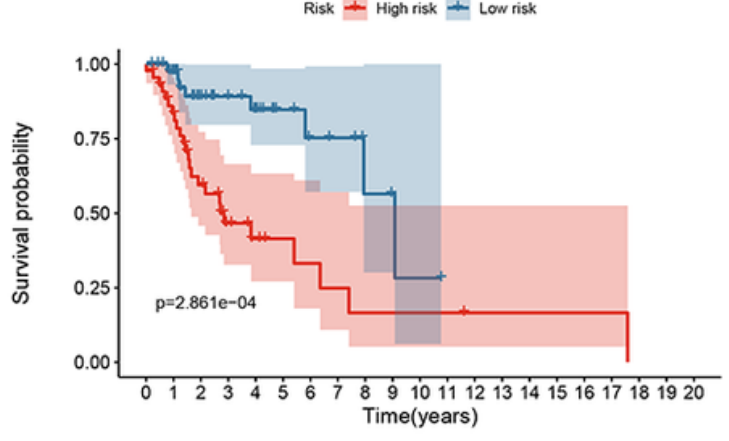

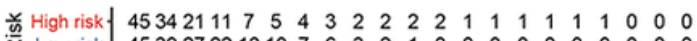

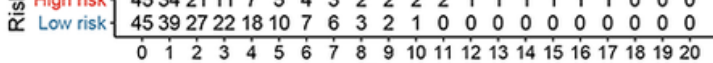
Time(years)

B ROC curve $(A U C=0.748)$

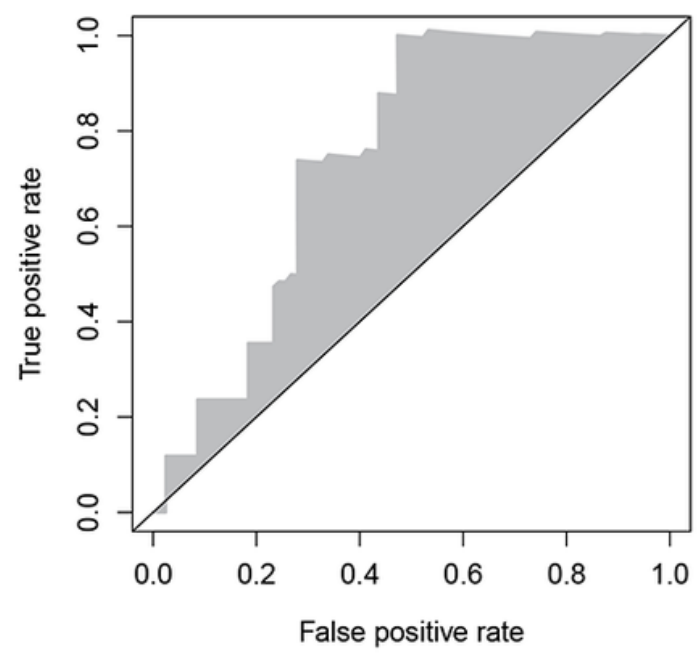

$\mathrm{C}$

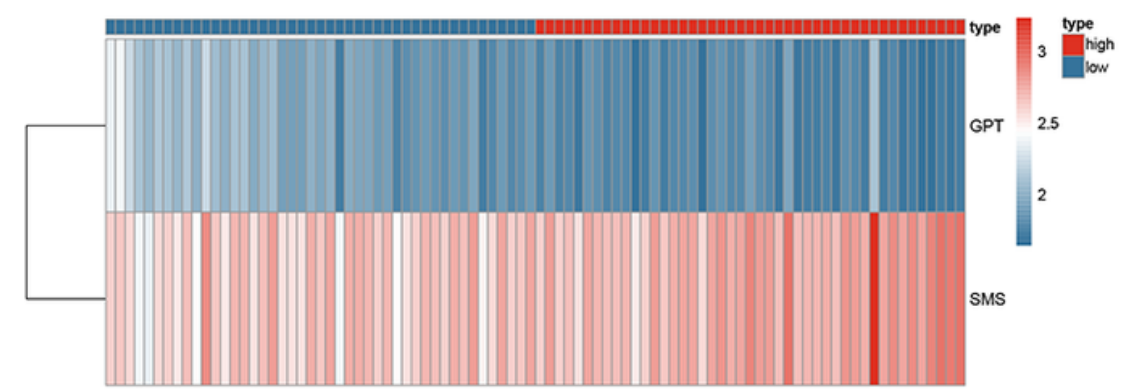

$\mathrm{D}$

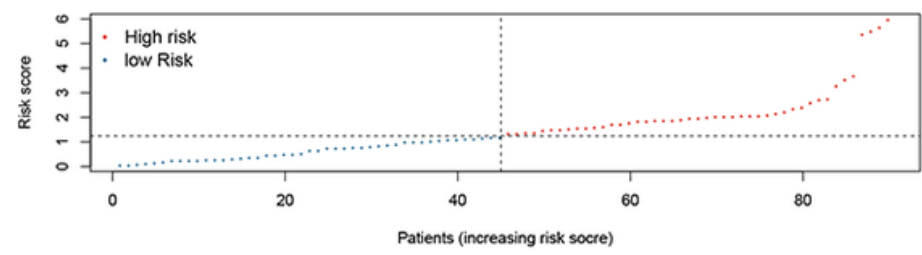

E

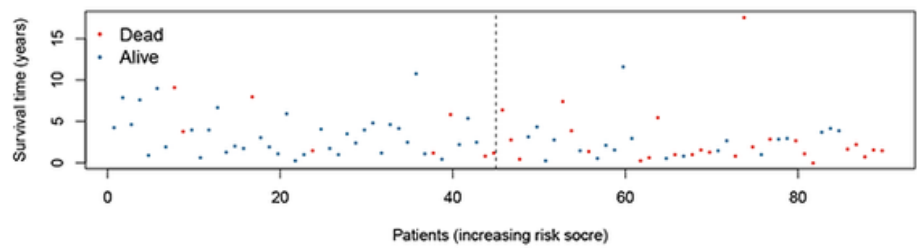

Figure 5

Internal validation of the prognosis signature in TCGA cohort. (A) KM survival analysis of high-risk and low-risk patients. (B) ROC curve of TCGA cohort. (C) Heatmap of GPT and SMS expression. (D, E) The survival status and risk score of patients. 
A

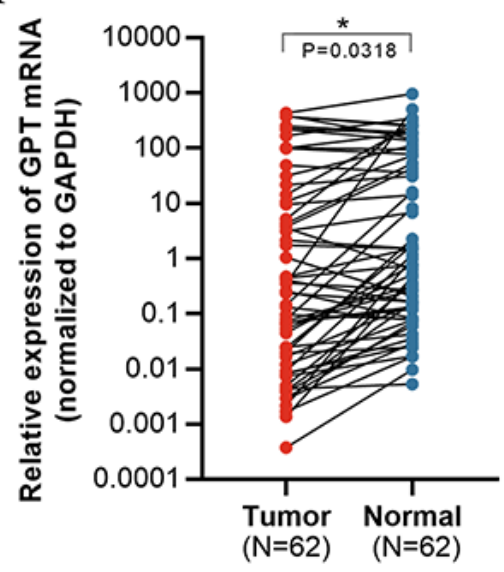

B

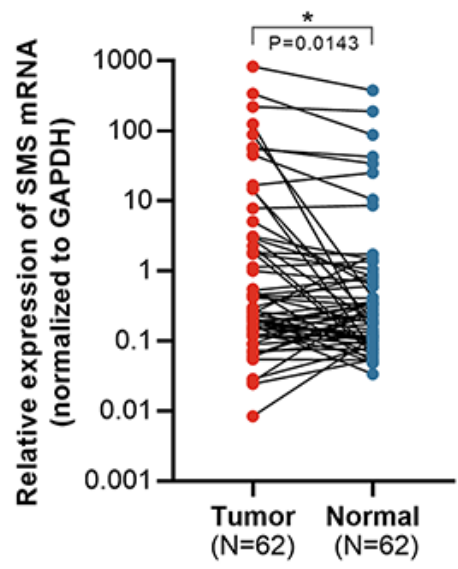

$\mathrm{C}$

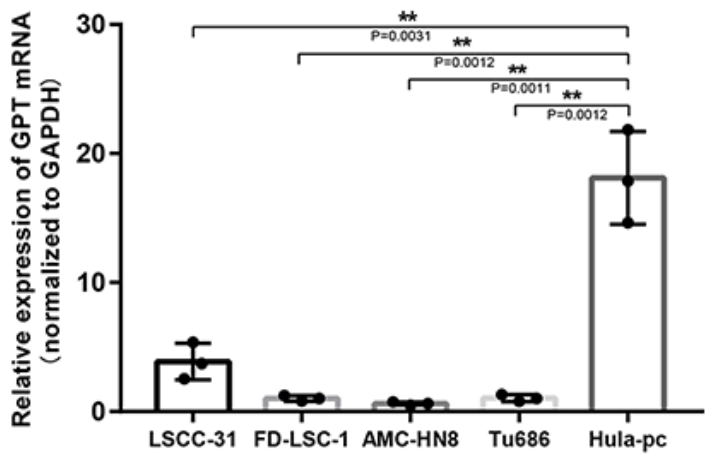

$\mathrm{D}$

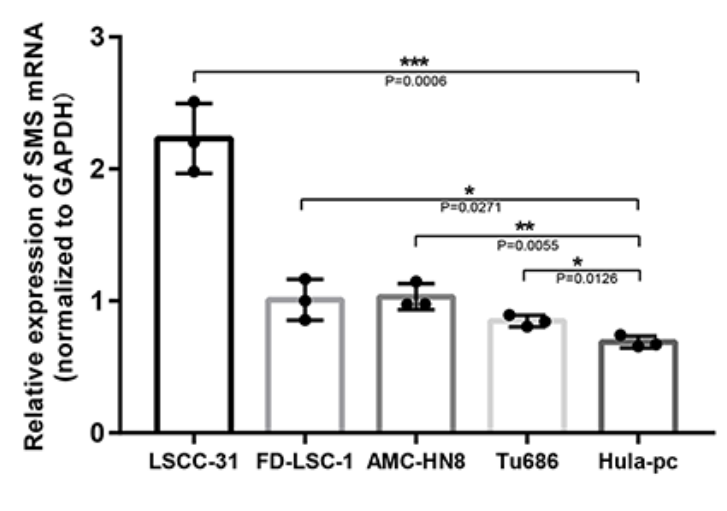

$\mathrm{E}$

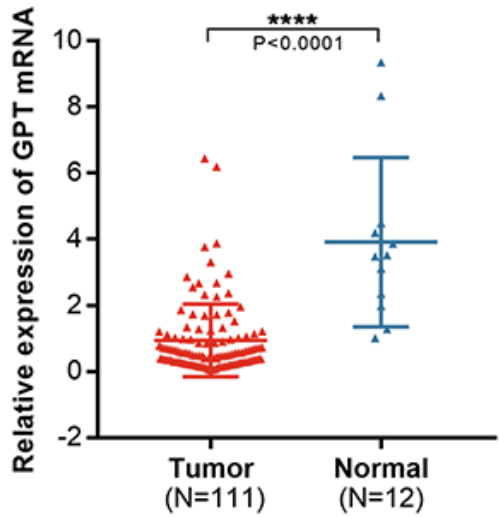

$\mathrm{F}$

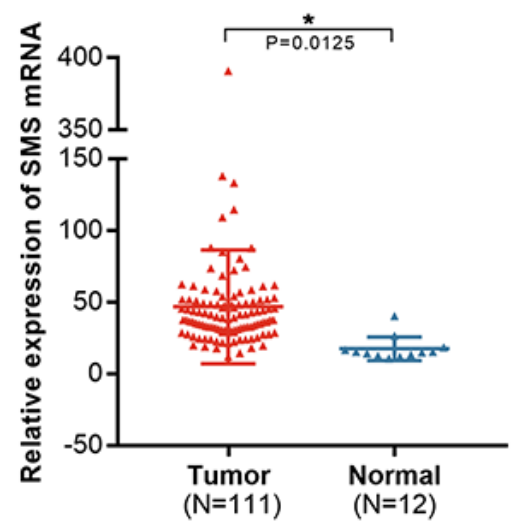

Figure 6

SMS and GPT expression in LSCC patients and cell lines. (A) GPT was down-regulated in LSCC tissues compared to adjacent normal tissues. (B) SMS was up-regulated in LSCC tissues compared to adjacent normal tissues. (C) GPT was down-regulated in LSCC cell lines to HuLa-PC, a cell line derived from posterior commissure of the larynx. (D) SMS was up-regulated in LSCC cell lines to HuLa-PC. (E) Relative expression of GPT in TCGA. (F) Relative expression of SMS in TCGA. 
A

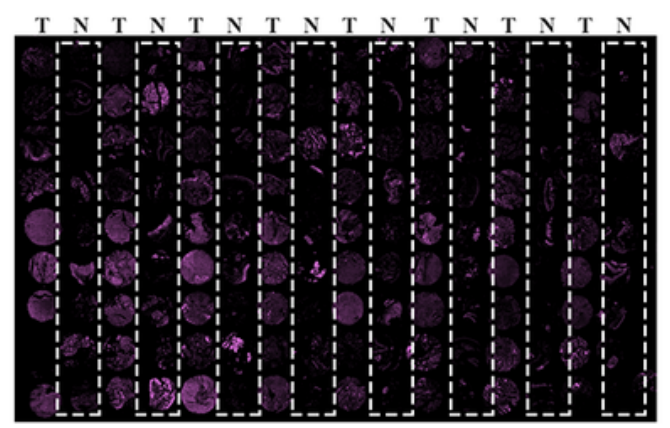

D

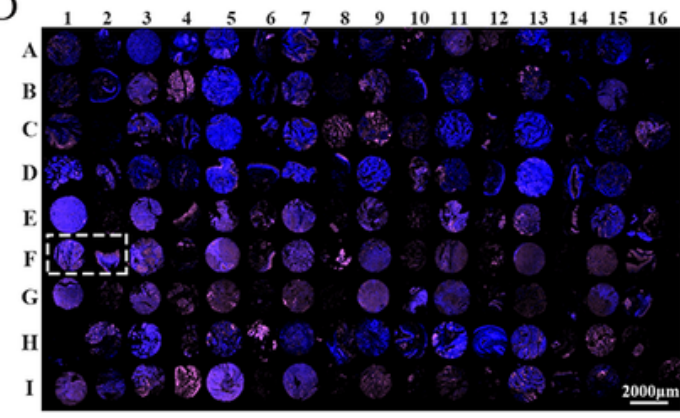

B

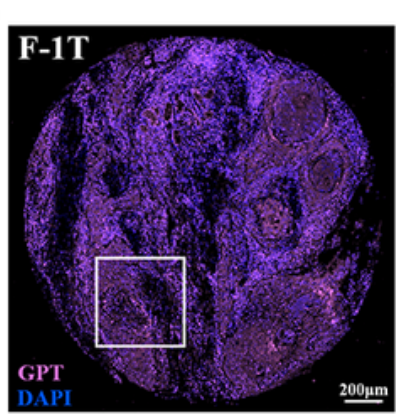

E

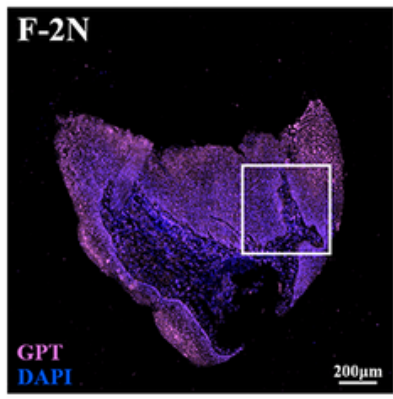

C

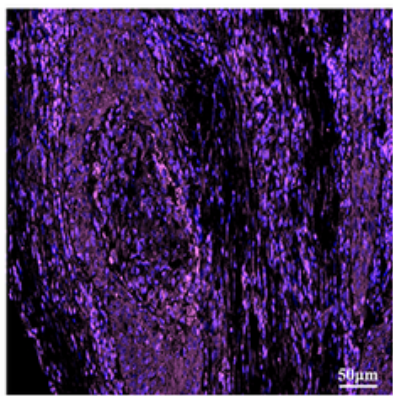

F

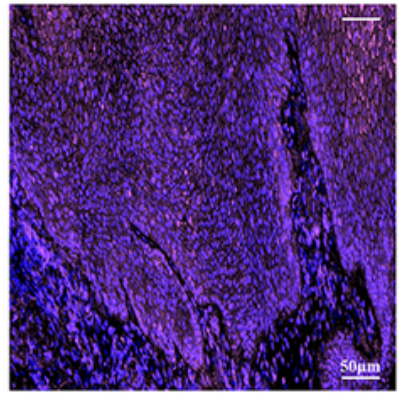

G

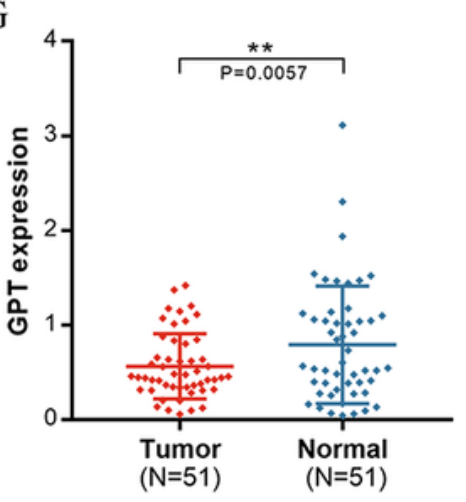

$\mathrm{J}$

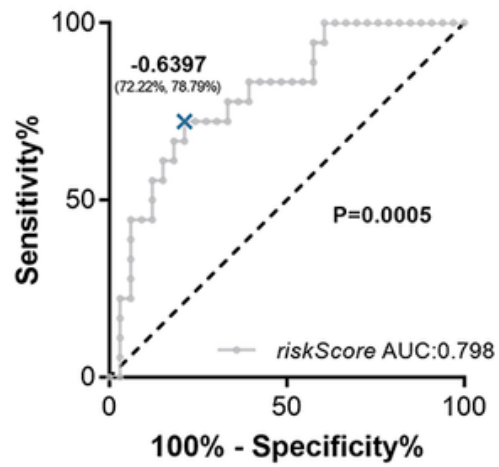

$\mathrm{H}$

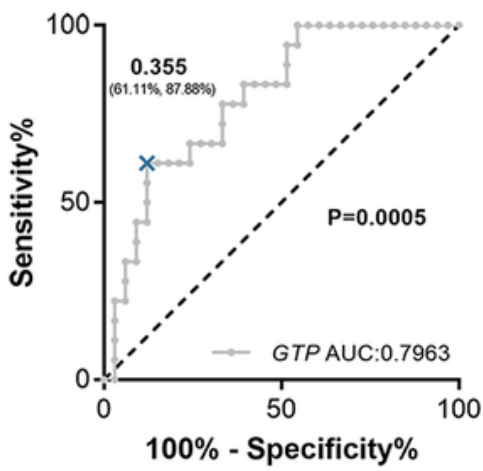

K
I

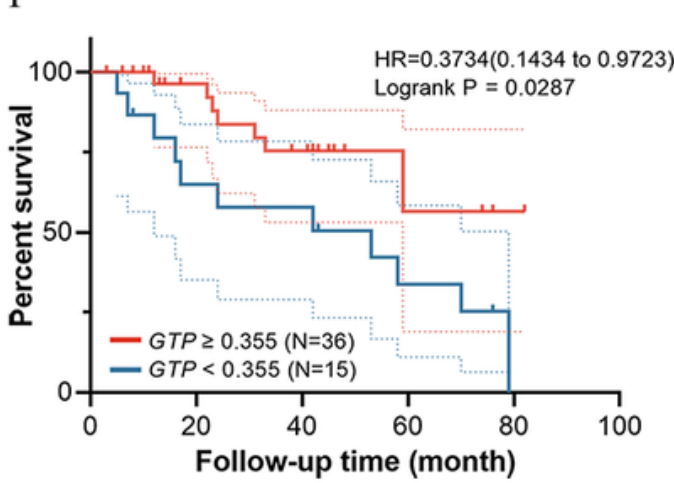

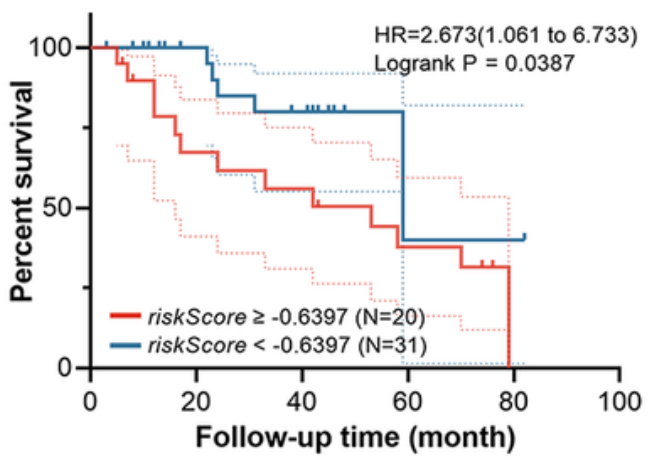

Figure 7

External validation of the prognosis signature in FDEENT cohort. (A) GPT inmmunofluorescence staining on TMA. (B, C) Merged inmmunofluorescence staining on F-1T. (D) Overview of merged inmmunofluorescence staining on TMA. (E, F) Merged inmmunofluorescence staining on F-2N. (G) Quantification of the immunofluorescence signals of GPT. (H) ROC curve of GPT prediction. (I) Survival 
analysis of GPT by the KM plotter in LSCC. (J) ROC curve of risk score (GPT+SMS) prediction. (K) Survival analysis of risk score (GPT+SMS) by the KM plotter in LSCC.

A Univariable risk factor for OS

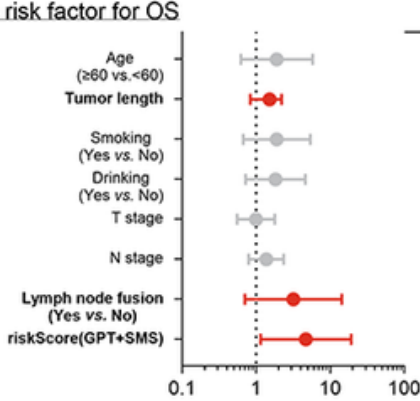

$\mathrm{C}$

Univariable risk factor for OS
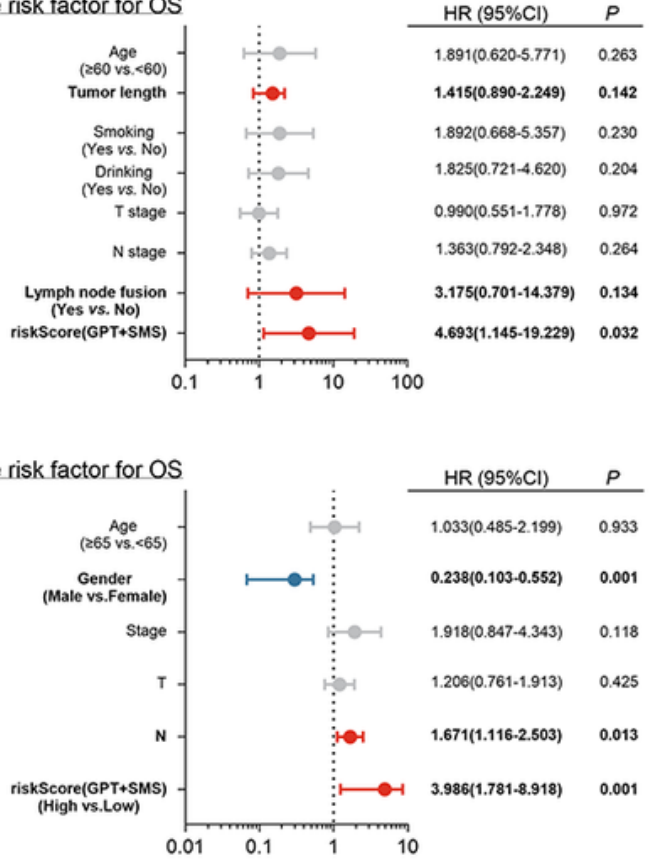

$\mathrm{C}$$$
\begin{array}{llll}
0.01 & 0.1 & 1 & 10
\end{array}
$$

B
Multivariable risk factor for OS

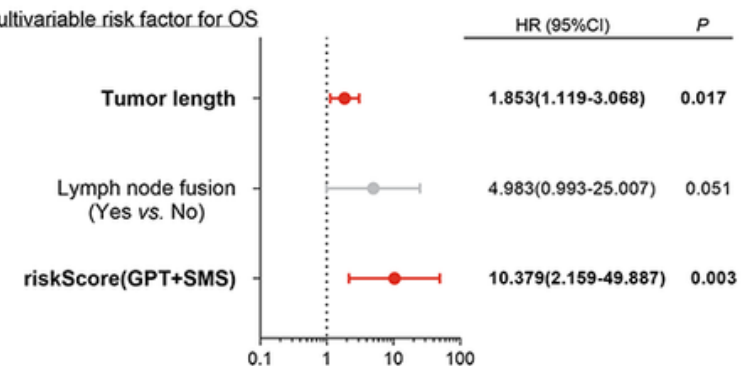

$\mathrm{D}$

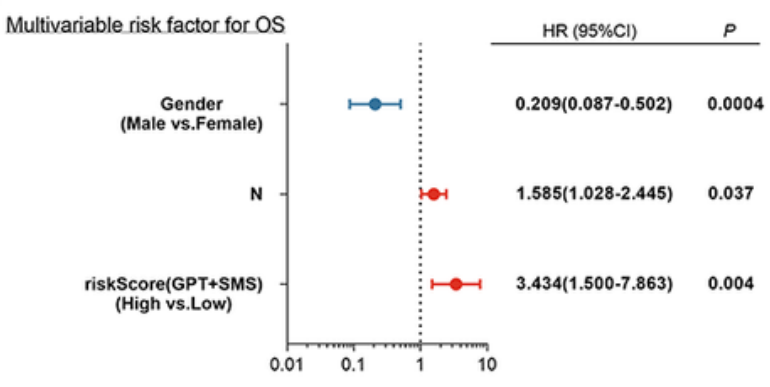

$\mathrm{E}$

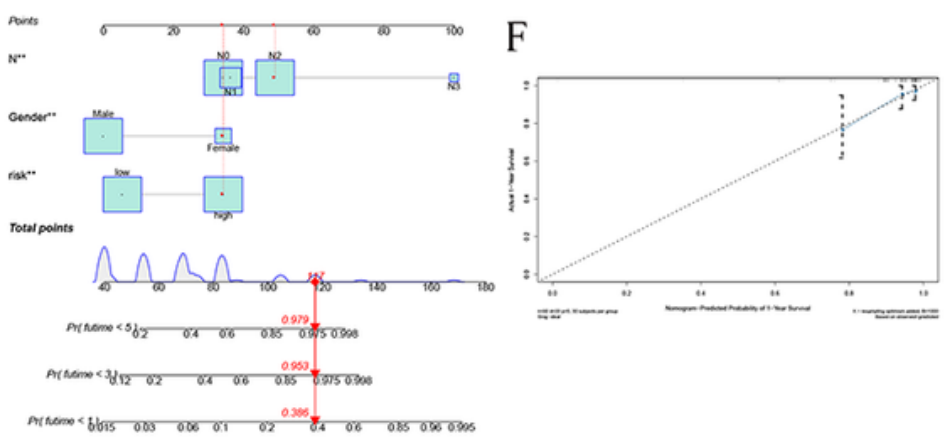

G $\quad \mathrm{H}$
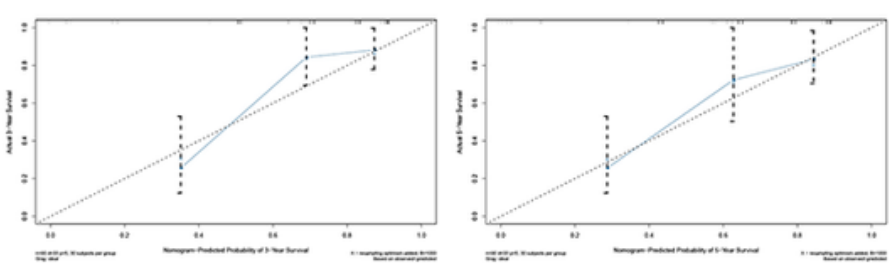

I

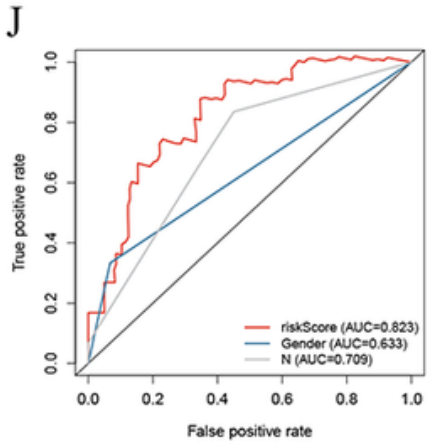

$\mathrm{K}$

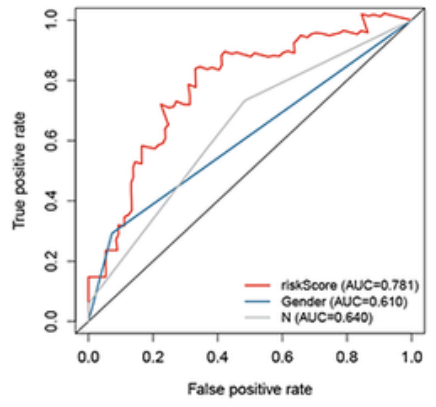

$\mathrm{L}$

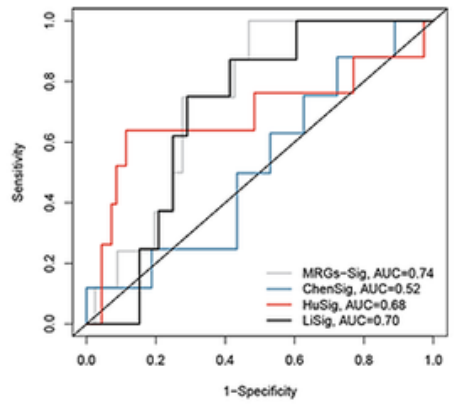

\section{Figure 8}

Independent prognostic analysis and construction of a nomogram generated from independent prognostic factors for predicting OS. (A, B) Results of univariate and multivariate Cox regression analysis of FDEENT cohort. (C, D) Results of univariate and multivariate Cox regression analysis of TCGA cohort. 
(E) The nomogram based on the independent prognostic factors of TCGA cohort. $(F, G, H)$ Calibration curve of prognostic signature for 1- , 3- , and 5- year's OS. (I, J, K) ROC curve of independent prognostic factors in 1-, 3- , and 5- year's. (L) Comparison of prognostic signature with other models.

\section{Supplementary Files}

This is a list of supplementary files associated with this preprint. Click to download.

- table1.xIsx

- table2.xIsx

- SupplementaryFigure1.tif

- SupplementaryFigure2.tif

- SupplementaryFigure3.tif

- SupplementaryFigure4.tif

- SupplementaryTable1.xlsx

- SupplementaryTable2.xlsx

- SupplementaryTable3.xlsx

- SupplementaryTable5.xlsx

- Supplementarytable4.xlsx 